\title{
Outcomes of Trauma Victims with Cardiac Arrest Who Survived to Intensive Care Unit Admission in a Level 1 Apex Indian Trauma Centre: A Retrospective Cohort Study
}

\author{
Kapil Dev Soni ${ }^{1} \oplus$, Nitin Rai $^{2} \oplus$, Richa Aggarwal ${ }^{3} \odot$, Anjan Trikha ${ }^{4} \odot$
}

\begin{abstract}
Background and aims: The prognosis of patients with cardiac arrest following trauma is poor. Our objectives were: (1) to determine outcomes of patients following in-hospital cardiac arrest posttrauma and admitted to the intensive care unit (ICU) and (2) to identify characteristics associated with in-hospital mortality.

Materials and methods: This was a single-center retrospective analysis of patients admitted to ICU after resuscitation following in-hospital cardiac arrest between January 2017 and July 2018. Patients with isolated head injuries and multiple cardiac arrests were excluded. Bivariate analysis was done to determine a significant association between baseline characteristics and in-hospital mortality.

Results: A total of 37 patients were included. About 35.1\% of trauma subjects survived hospital discharge. Bivariate analysis showed positive association between admission Acute Physiology and Chronic Health Evaluation II (APACHE II) and Sequential Organ Failure Assessment (SOFA) scores with in-hospital mortality. Other characteristics, such as age, duration of cardiopulmonary resuscitation (CPR), and serum lactate levels on admission, were not associated with in-hospital mortality.

Conclusion: Despite being at lower survival following a cardiac arrest after trauma, approximately one-third of the patients survived hospital discharge. This implies that aggressive support of this population is not necessarily futile. Optimization of postresuscitation physiological factors and their impacts on outcomes for these patients need further studies.
\end{abstract}

Keywords: In-hospital cardiac arrest, Outcomes, Prognostic factors.

Indian Journal of Critical Care Medicine (2021): 10.5005/jp-journals-10071-24057

\section{INTRODUCTION}

Patients suffering from trauma-related cardiac arrest (TRCA) are generally reported to have a poor outcome, ${ }^{1,2}$ and arguments for the futility of aggressive support have been made for this patient population. ${ }^{3,4}$ More recent publications, using data from, have reported that TRCA survival rates are comparable to out-of-hospital medical cardiac arrest according to a prospective registered trauma system database. ${ }^{5}$ Patients with TRCA have a similar prognosis compared with atraumatic cardiac arrest (ATCA) patients; however, most of the subjects in this study did not have a return of spontaneous circulation. ${ }^{6}$ An epidemiological study conducted in India showed that patients presented with TRCA, either out-ofhospital cardiac arrest (OHCA) or in emergency department (ED), who were provided cardiopulmonary resuscitation (CPR), return of spontaneous circulation (ROSC) was present in $7.4 \%$ and $92.60 \%$ of the patients could not be revived. ${ }^{7}$

There is a paucity of literature in the Indian scenario, answering the factors associated with survival to hospital discharge of trauma victims with postcardiac arrest surviving to intensive care unit (ICU) admission.

In our study, we propose to determine the factors related to outcomes for patients following in-hospital cardiac arrest posttrauma and admitted to the ICU and to identify characteristics associated with in-hospital mortality. This will help optimize the postresuscitation parameters that could improve outcomes for patients resuscitated after TRCA.
1,3 Department of Critical and Intensive Care, JPN Apex Trauma Centre, All India Institute of Medical Sciences, New Delhi, India

${ }^{2}$ Department of Critical Care Medicine, KGMU, Lucknow, Uttar Pradesh, India

${ }^{4}$ Department of Anaesthesiology, All India Institute of Medical Sciences, New Delhi, India

Corresponding Author: Richa Aggarwal, Department of Critical and Intensive Care, JPN Apex Trauma Centre, All India Institute of Medical Sciences, New Delhi, India, Phone: +91 9873731042, e-mail: pathakricha@yahoo.co.in

How to cite this article: Soni KD, Rai N, Aggarwal R, Trikha A. Outcomes of Trauma Victims with Cardiac Arrest Who Survived to Intensive Care Unit Admission in a Level 1 Apex Indian Trauma Centre: A Retrospective Cohort Study. Indian J Crit Care Med 2021;25(12):1408-1412.

Source of support: Nil

Conflict of interest: None

\section{Methods}

Institute Ethics Committee clearance was obtained prior to the study (Ref. No. IEC-155/05.04.2019). This was a retrospective single-centered observational study analyzing data of the patients admitted to Level 1 Apex Trauma Centre ICU following in-hospital cardiac arrest between January 2017 and July 2018. Details of the patients arriving to ED were retrieved from the

(o) The Author(s). 2021 Open Access This article is distributed under the terms of the Creative Commons Attribution 4.0 International License (https://creativecommons. org/licenses/by-nc/4.0/), which permits unrestricted use, distribution, and non-commercial reproduction in any medium, provided you give appropriate credit to the original author(s) and the source, provide a link to the Creative Commons license, and indicate if changes were made. The Creative Commons Public Domain Dedication waiver (http://creativecommons.org/publicdomain/zero/1.0/) applies to the data made available in this article, unless otherwise stated. 
paper-based registers/ICU registry/hospital medical records section retrospectively. The inclusion criteria were postcardiac arrest patients following trauma and being admitted to polytrauma ICU following resuscitation. Patients with isolated head injuries and multiple cardiac arrests were excluded. Data retrieval was done from the hospital medical record section. Data were screened and the following covariables and factors (measured as categorical and continuous) were recordedduration of CPR, mechanism of injury, place of cardiac arrest, cause of cardiac arrest and Glasgow coma scale (GCS) on arrival to ICU, vasopressors/inotropes requirement and duration of blood gas parameters, ventilator settings, indication for ICU admission, hemodynamic parameters, urine output, and severity scoring-Acute Physiology and Chronic Health Evaluation II (APACHE II) and Sequential Organ Failure Assessment (SOFA).

\section{Results}

A total of 37 patients were included in the study. Out of these, 31 $(83.8 \%)$ were male and 6 (16.2\%) were female. Long bone fracture followed by blunt trauma abdomen (BTA) and blunt trauma chest (BTC) were the most common modes of injury contributing 24.2 and $18.2 \%$, respectively. Emergency areas and wards were the most common sites of cardiac arrest ( $40.2 \%$ each) (Fig. 1). Cause of cardiac arrest could be ascertained in 20 cases, as enlisted in Figure 2.

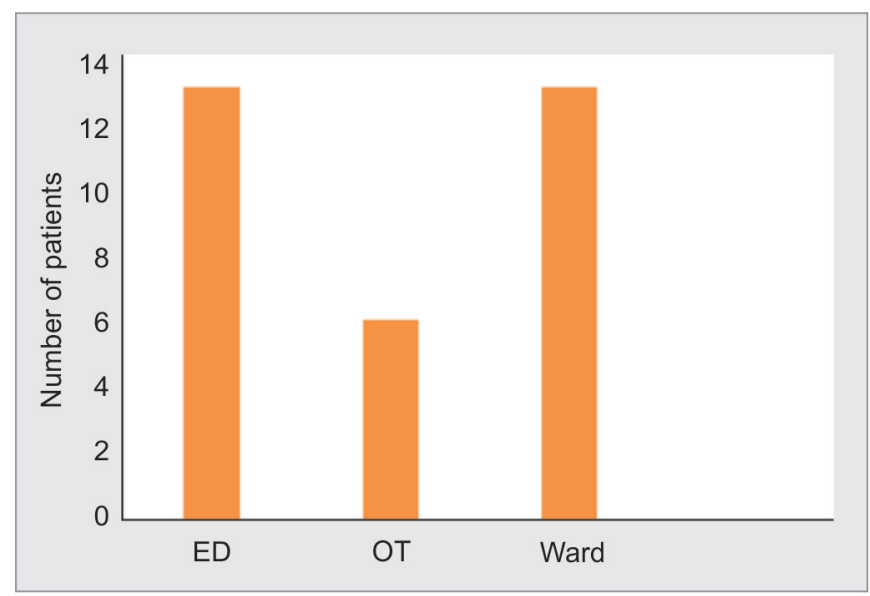

Fig. 1: Sites of cardiac arrest

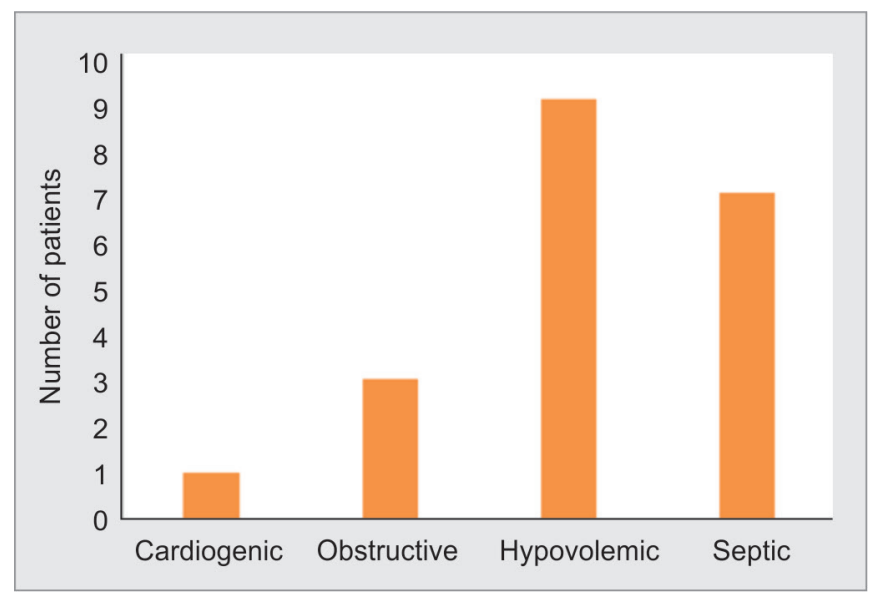

Fig. 2: Causes of cardiac arrest

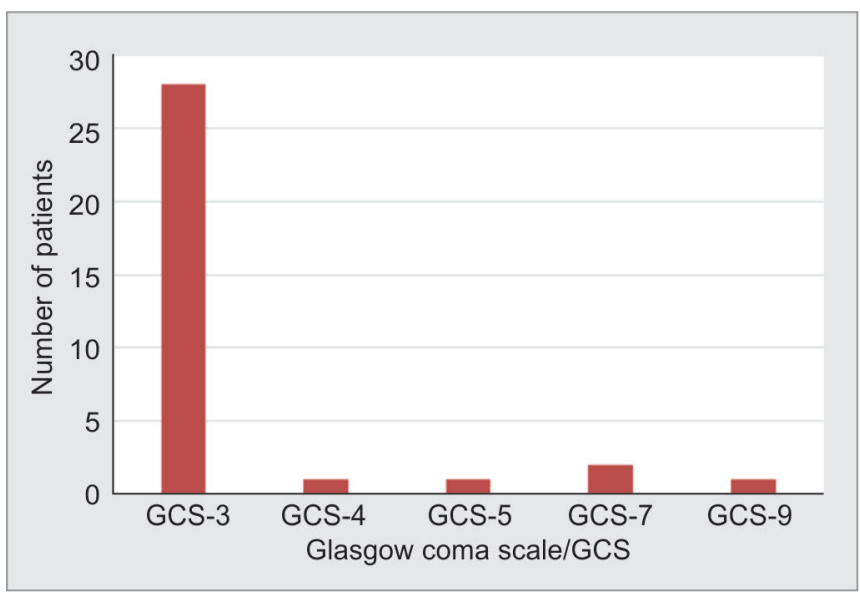

Fig. 3: Glasgow coma scale (GCS) on ICU admission

The mean duration of CPR was 22.7 minutes, with a range of a minimum of 5 to a maximum of 45 minutes.

In the majority of the patients, GCS on admission to ICU was three (75.7\%) (Fig. 3).

A total of 31 out of 37 patients required vasopressors on ICU admission. The requirement was high (more than $20 \mu \mathrm{g} /$ minute) in $26(83.9 \%)$ cases and moderate (less than $20 \mu \mathrm{g} / \mathrm{min}$ ) in $6(16.1 \%)$ cases. The mean duration of vasopressor days was 4.9 days, with a minimum and maximum of 1 and 45 days, respectively. The mean APACHE II and SOFA scores were 25.8 and 12.8 , respectively (Figs $4 \mathrm{~A}$ and $\mathrm{B}$ ).

The average ICU stay and hospital length of stay were 4.9 and 6.1 day, respectively. A total of $35.1 \%$ of the patients survived to hospital discharge. APACHE II and SOFA within the first 24 hours were associated significantly with in-hospital mortality in bivariate analysis.

*0 represents survivors and 1 represents death

13 out of 37 (35.1\%) patients survived to hospital discharge.

\section{Discussion}

This study was an attempt to elucidate the factors associated with the outcomes following in-hospital cardiac arrests. Most of the studies have analyzed OHCAs or cardiac arrests occurring in the ED. ${ }^{5-7}$

A total of 13 out of 37 (35.1\%) patients survived to hospital discharge. Traditionally, survival after cardiac arrest is generally considered abysmal. There has been a long-term debate over for futility of aggressive support for this patient population. ${ }^{3,4}$ There has been no direct analysis of survival to hospital discharge following in-hospital cardiac arrest. Girotra et al. ${ }^{8}$ analyzed the trends in survival following in-hospital cardiac arrest. They concluded that risk-adjusted rates of survival to discharge were $13.7-22.3 \%$ over the 9 years of observation period. David et al. ${ }^{6}$ compared the outcome of patients after traumatic or atraumatic ("medical") OHCA. There was no significant difference between traumatic or atraumatic groups at hospital discharge [2.2\% (0.5-4.0\%) vs $2.8 \%(2.1-3.4 \%)]$. Chen et al. ${ }^{9}$ in a recently conducted study reported a 30 -day survival of $14 \%$. Significantly better survival to discharge rate in the present study could be explained by two attributes. First, being a witnessed arrest inside the hospital, there was a timely and quality initiation of resuscitative efforts by medical personnel, unlike in OHCA. This 

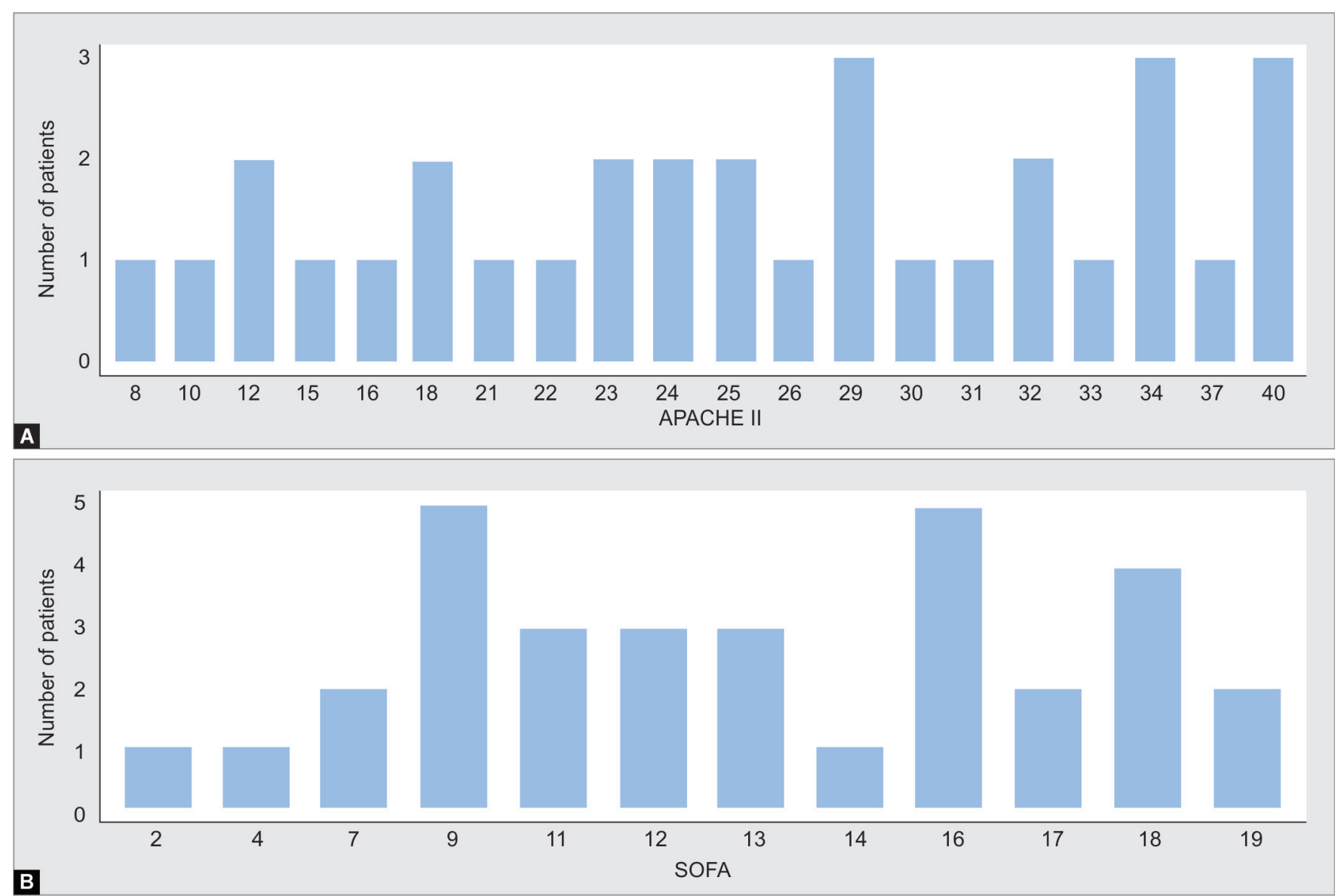

Figs 4A and B: (A) APACHE II score distribution; (B) SOFA score distribution
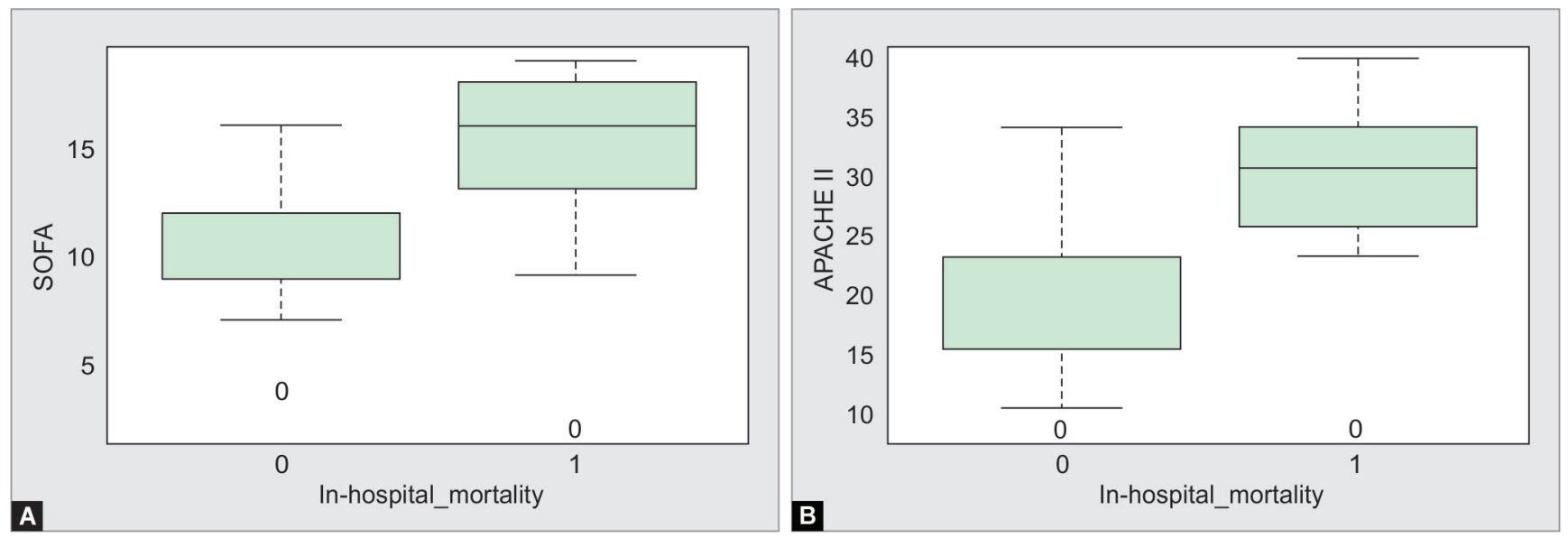

Figs 5A and B: Box plot describing distribution of disease severity between survivors and dead patients. Within each box, horizontal dark lines denote median values; boxes extend from the 25 th to the 75 th percentile of each group's distribution of values; vertical extending lines denote adjacent values (i.e., the most extreme values within 1.5 interquartile range of the 25 th and 75 th percentile of each group)

was a major contributor to better outcomes. Second, a small sample size could have produced an inflated result.

Severity scores-APACHE II and SOFA-within the first 24 hours significantly associated with in-hospital mortality in bivariate analysis (Figs 5 and 6) whereas age, duration of CPR, and serum lactate levels were not associated with in-hospital mortality
(Fig. 7). Physiologic predictors for survival in the posttraumatic arrest were analyzed by Cera et al. ${ }^{2}$ They concluded that sinus rhythm and pupil size/reactivity are important parameters that predicted potential survival and may be used as a guide for the continuation of resuscitative efforts. Similarly, Chen et al. ${ }^{9}$ evaluated factors associated with outcomes in traumatic cardiac 


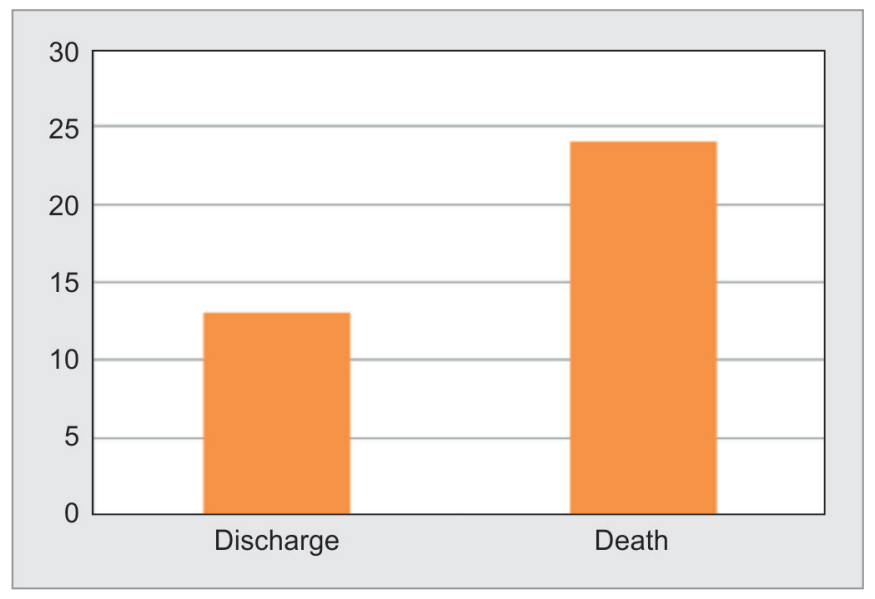

Fig. 6: Hospital outcome
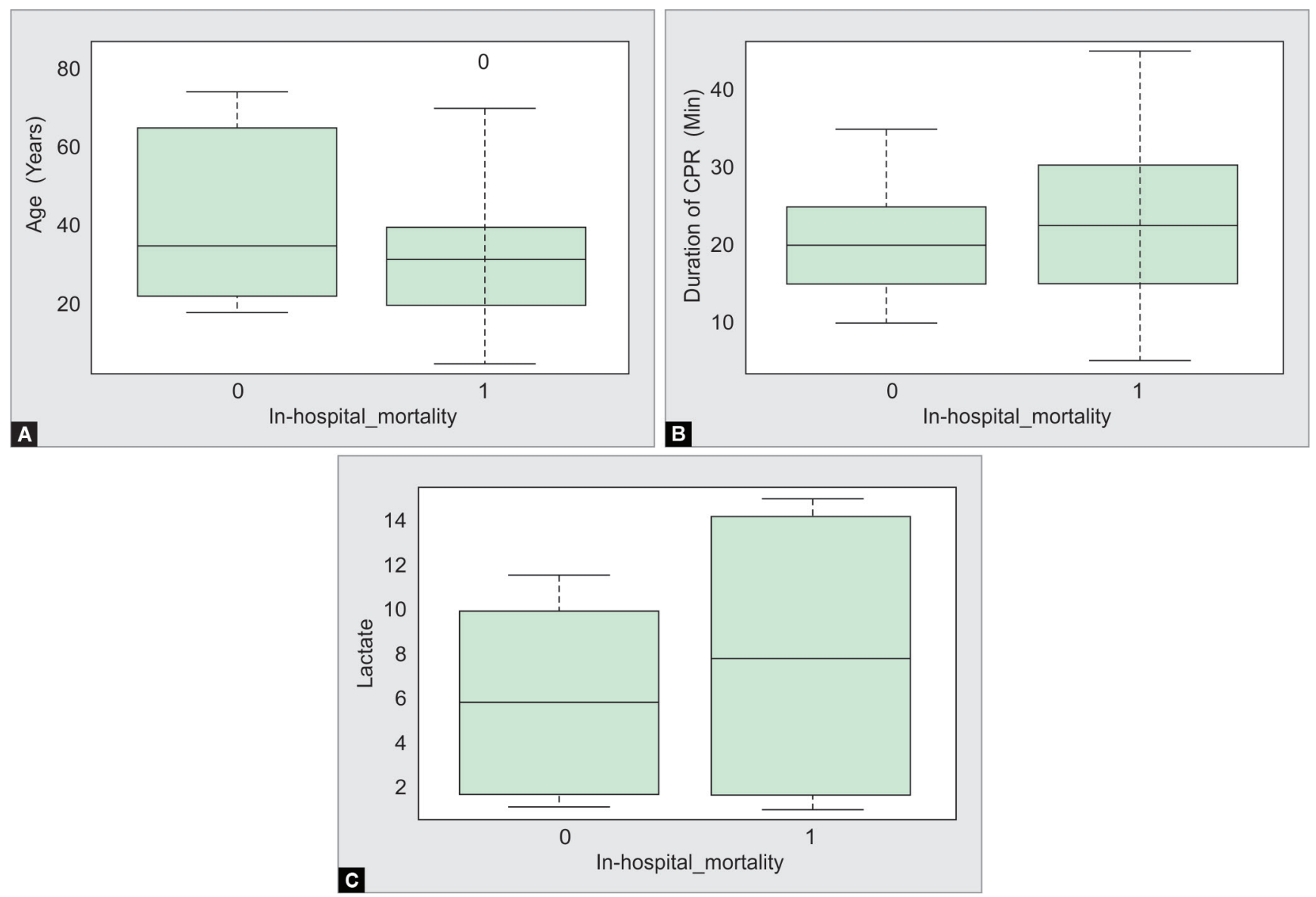

Figs 7A to C: Box plot describing distribution of age, duration of CPR, and lactate level between survivors and dead patients. Within each box, horizontal dark lines denote median values; boxes extend from the 25th to the 75th percentile of each group's distribution of values; vertical extending lines denote adjacent values (i.e., the most extreme values within 1.5 interquartile range of the 25 th and 75 th percentile of each group)

arrest patients without prehospital return of spontaneous circulation. In their study, Injury Severity Score of $\geq 16(\mathrm{OR}, 0.06$; $95 \% \mathrm{Cl}: 0.02-0.20)$, length of ED resuscitation (OR, 0.98; $95 \%$ $\mathrm{Cl}: 0.96-0.99)$, trauma center admission (OR, 2.69; 95\% Cl: 1.03-7.03), and total resuscitation length of $>20$ minutes (OR, $0.21 ; 95 \% \mathrm{Cl}$ : 0.08-0.54) were associated with ROSC.
It can be concluded that resuscitative efforts should be continued despite previous reports of terminating over futility as a survival to discharge rate in one-third of patients is quite significant considering the perspectives of previous data. Investigation into whether optimization of postresuscitation factors would improve the outcome for these patients may be warranted. 


\section{OrCID}

Kapil Dev Soni @ https://orcid.org/0000-0003-1214-4119

Nitin Rai 구 https://orcid.org/0000-0003-3351-4553

Richa Aggarwal @ https://orcid.org/0000-0002-4531-2759

Anjan Trikha $\odot$ https://orcid.org/0000-0002-6001-8486

\section{References}

1. Battistella FD, Nugent W, Owings JT, Anderson JT. Field triage of the pulseless trauma patient. Arch Surg 1999;134(7):742-745; discussion 745-746. DOI: 10.1001/archsurg.134.7.742.

2. Cera SM, Mostafa G, Sing RF, Sarafin JL, Matthews BD, Heniford BT. Physiologic predictors of survival in post-traumatic arrest. Am Surg 2003;69:140-144. PMID: 12641355.

3. Hopson LR, Hirsh E, Delgado J, et al. Guidelines for withholding or termination of resuscitation in prehospital traumatic cardiopulmonary arrest: joint position statement of the National Association of EMS Physicians and the American College of Surgeons Committee on Trauma. J Am Coll Surg 2003;196(1):106-112. DOI: 10.1016/s 1072-7515(02)01668-x.

4. Stockinger ZT, McSwain NE Jr. Additional evidence in support of withholding or terminating cardiopulmonary resuscitation for trauma patients in the field. J Am Coll Surg 2004;198(2):227-231. DOI: 10.1016/j.jamcollsurg.2003.10.012.

5. Evans CC, Petersen A, Meier EN, Buick JE, Schreiber M, Kannas D, et al. Resuscitation outcomes consortium I. Prehospital traumatic cardiac arrest: management and outcomes from the resuscitation outcomes consortium epistry-trauma and PROPHET registries. J Trauma Acute Care Surg 2016;81(2):285-293. DOI: 10.1097/ TA.0000000000001070.

6. David JS, Gueugniaud PY, Riou B, Pham E, Dubien PY, Goldstein P, et al. Does the prognosis of cardiac arrest differ in trauma patients? Crit Care Med 2007;35(10):2251-2255. DOI: 10.1097/01. ccm.0000281859.61545.22.

7. Bhoi S, Mishra PR, Soni KD, Baitha U, Sinha TP. Epidemiology of traumatic cardiac arrest in patients presenting to emergency department at a level 1 trauma center. Indian J Crit Care Med 2016;20(8):469-472. DOI: 10.4103/0972-5229.188198.

8. Girotra S, Nallamothu BK, Spertus JA, Li Y, Krumholz HM, Chan PS, et al. Trends in survival after in-hospital cardiac arrest. N Engl J Med 2012;367(20):1912-1920. DOI: 10.1056/NEJMoa1109148.

9. Chen YC, Wu KH, Hsiao KY, Hung MS, Lai YC, Chen YS, et al. Factors associated with outcomes in traumatic cardiac arrest patients without prehospital return of spontaneous circulation. Injury 2019;50(1):4-9. DOI: 10.1016/j.injury.2018.07.010. 